\title{
Assessing Relative Importance Using RSP Scoring to Generate Variable Importance Factor (VIF)
}

\author{
Daniel Koh ${ }^{1}$ \\ ${ }^{1}$ School of Business, SIM University, Singapore \\ Correspondence: Daniel Koh, School of Business, SIM University, 461 Clementi Road, 599 491, Singapore. Tel: \\ 65-6248-9746. E-mail: danielkoh005@unisim.edu.sg
}

\author{
Received: March 17, 2015 Accepted: April 8, 2015 Online Published: April 27, 2015 \\ doi:10.5539/ijsp.v4n2p123 URL: http://dx.doi.org/10.5539/ijsp.v4n2p123
}

\begin{abstract}
Previous research has shown that the construction of VIF is challenging. Some researchers have sought to use orderly contribution of $R^{2}$ (coefficient of determination) as measurement for relative importance of variable in a model, while others have sought the standardized parameter estimates $b$ (beta) instead. These contributions have been proven to be very valuable to the literature. However, there is a lack of study in combining key properties of variable importance into one composite score. For example, an intuitive understanding of variable importance is by scoring reliability, significance and power (RSP) of it in the model. Thereafter the RSP scores can be aggregated together to form a composite score that reflects VIF. In this paper, the author seeks to prove the usefulness of the DS methodology. DS stands for Driver's Score and is defined as the relative, practical importance of a variable based on RSP scoring. An industry data was used to generate DS for practical example in this paper. This DS is then translated into a $2 \times 6$ matrix where level of importance $(L x I)$ is generated. The final outcome of this paper is to discuss the use of RSP scoring methodology, theoretical and practical use of DS and the possible future research that entails this paper. DS methodology is new to the existing literature.
\end{abstract}

Keywords: variable importance, decomposition of variances, RSP Scoring, multiple linear regression

\section{Introduction}

In recent history, much effort has been given to the study of variable importance factor (VIF). Researchers have approached this topic in several manners: taking the increase of $R^{2}$ - coefficient of determination - as the usefulness of the regressors (Darlington, 1968), squared standardized coefficients and products of standardized coefficients with marginal correlations (Hoffman, 1960; Hooker \& Yule, 1906), LMG method of using sequential sums of squares from the linear model (Lindeman, Merenda, \& Gold, 1980), conditional variable importance for Random Forest (Strobl, Boulesteix, Kneib, Augustin, \& Zeileis, 2008), the averaging method of variance decomposition (Kruskal, 1987; Chevan \& Sutherland, 1991) and proportional marginal variance decomposition. However, most of the studies are founded on one dimension. Several authors which include Ehrenberg (1990), Stufken (1992), and Christensen (1992) have dismissed the usefulness and benefits of relative importance measure. The premise of this dismissal was that the decomposition of coefficient of determination is too simplistic and it is difficult to tease out relative importance among correlated variables which could potentially "double count" in the model. In this paper, the focus is in the independent measurement of relative importance and the discussion on teasing out interrelatedness between independent variables is deferred.

The decomposition of coefficient of determination becomes a powerful tool when complementary scorings are given to improve accuracy in understanding relative importance of variables. Hence, this paper seeks to suggest a new method to assess relative importance of variable by considering reliability, significance and power, to the end that the final composite scores reflect the intuitive, practical understanding of relative importance better. Reliability is defined here as the invert of the sum of residual errors between the predicted and actual values. Significance is defined here as the heterogeneity of groupings with homogeneity in within-group distances or maximized distances between predicted values with expected mean of dependent variable and minimized distances between predicted values with actual values. Power is defined here as the positivity of the slope of the estimates, with greater or steeper slope leading to greater power. The intention is to develop a score that not only accounts for variance decomposition, but also practical meaningfulness and accuracy in utilizing a variable. It also accounts for the goodness of a predictor by scoring the standardized parameter estimates of the variable in 
the model. The DS scoring methodology is new to our existing literature today.

\section{Residual Errors as First Property}

One aspect of a good predictor is its minimized residual error values, which then contribute to a strong coefficient of determination, $\mathrm{R}^{2}$. When residual errors are minimized, the chance of making mispredictions becomes lesser, leading to greater reliability for the predictor. A practical example would be the reliability of age in understanding income earnings. Between the independent variable age and gender, age is chosen to be the better predictor for income earnings because gender may not contribute as much between-group residual errors as compared to the sum of squares of model (SSM) for age. This is particularly true when a meritocratic society promotes merit for work experiences, of which only age most likely correlates positively with it strongly, regardless of gender type. Intuitively, the concept of reliability lies on the confidence of which one can get when the model is put to test. The scores can be decomposed into respondent level, whereby each respondent is given three scores for RSP, leading up to the final scoring of DS.

Hence, the first function of DS is the invert of residual errors, which is first expressed in the following mathematical expression for multiple linear regression:

$$
\hat{\mathrm{y}}=a_{0}+a_{1} x_{1}+a_{2} x_{2}+\ldots+a_{n} x_{n}+\varepsilon
$$

where $x \in \mathbb{R}$ denotes the regressor, $\hat{y} \in \mathbb{R}$ denotes the dependent variable, $a$ denotes the parameter estimate and $\varepsilon$ denotes the error term of the model.

A series of $x$ is fitted into the model, generating a series of predicted values, $\hat{y}$. The residual for this model $-\zeta$ is then expressed as the absolute difference between the actual values of the dependent variable which is denoted by $y$ and the predicted values $\hat{y}$.

$$
\zeta=|y-\hat{y}|
$$

The residual is then inverted to form common directionality with two other functions of the RSP framework.

$$
\rho=\frac{1}{\zeta}=\frac{1}{|y-\hat{y}|}
$$

The residual is then fitted onto the Gaussian's cumulative distribution function - $\Phi(\rho)$ - , assuming that the variable is independent and identically distributed (I.I.D) under the Normal distribution, $X \sim N(0,1)$ :

$$
\Phi(\rho)=\frac{1}{\sigma \sqrt{2 \pi}} \int_{\min \rho}^{\rho} e^{-\left(\rho-\mu_{\rho}\right)^{2} / 2 \sigma_{\rho}^{2}} d \rho
$$

where $\rho$ denotes the inverse of residual error, $\mu_{\rho}$ denotes the mean of the residual errors, $\sigma_{\rho}$ denotes the standard deviation of the residual error.

The first function of DS is improved when $\sigma^{2}{ }_{\rho}$ is decreased: reliability increases when data are less sparsely distributed. This cumulative distribution function serves as a score for reliability on the observation or respondent level. The use of the magnitude of $R^{2}$ contributions to assess relative importance was similarly proposed by Hoffman (1960) and later defended by Pratt (1987).

\section{F-Ratios of the Residual Errors as Second Property}

While the residual errors are expected to be minimized, the significance of the residual errors is expected to be maximized. The motivation behind this property is to obtain scores that reflect variable's distinctiveness among inter-groups through a study of variance ratio. For example, if a variable $\lambda$ has unique and distinctive $\kappa$ groupings in understanding income earning levels of a country, the F-Ratios due to $\lambda$ should be greater as compared to those that have more homogenous groupings.

The residual error in the first function is preferred over observations because the variance of residual errors (variance study of residual errors) is expected to reflect distinctiveness between groups better if they are truly 
distinctive and unique than the observation values themselves. For example, when income is predicted using Age, the error between $\kappa$ groupings in $\lambda$ should have distinctive noises. This "distinctive noises" should characterize their identity as unique groupings in the model. This is true for variables that are categorical, which will partition the data into $k$ th groupings. Hence, the second function of DS has an inverse relationship with the first function of DS.

The second function of DS - $\rho$ - is expressed in the following mathematical expression for a linear regression model:

$$
F_{\rho}=\frac{\text { Variances due to Model }}{\text { Variances due to Residual }}
$$

$$
\begin{gathered}
F_{\rho}=\frac{\frac{\sum(\hat{y}-\bar{y})^{2}}{(K-1)}}{\frac{\sum(y-\hat{y})^{2}}{(N-K)}} \\
F_{\rho}=\frac{\sum(\hat{y}-\bar{y})^{2}}{\sum(y-\hat{y})^{2}}\left(\frac{N-K}{K-1}\right)
\end{gathered}
$$

where $K$ is the number of groupings, $\hat{y}$ denotes predicted value of $y$, and $\bar{y}$ denotes the average value of $y$, $N$ denotes the sample size.

The F-Ratios are then fitted into Fisher's CDF, which is the integral of the PDF of F-distribution, assuming that the variables are independent and identically distributed (I.I.D) under the Fisher's distribution:

$$
\begin{gathered}
\Phi_{\mathrm{F}_{\rho}\left(\mathrm{F}_{\rho}, K-1, N-K\right)}=\int_{0}^{\mathrm{F}_{\rho}} \frac{\left(\frac{K-1}{N-K}\right)^{\frac{K-1}{2}} \mathrm{~F}_{\rho}^{\frac{K-1}{2}-1}\left(1+\frac{K-1}{N-K} \mathrm{~F}_{\rho}\right)^{-\frac{\mathrm{N}-1}{2}}}{\left[\frac{\left[\frac{1}{2}(N-K)\right] !\left(\frac{1}{2}(K-1)\right] !}{\left[\frac{1}{2}(N-K)\right]+\left[\frac{1}{2}(K-1)\right]-1}\right]} \\
\Phi_{Q}=1-\int_{0}^{\mathrm{F}_{\rho}} \frac{\left(\frac{K-1}{N-K}\right)^{\frac{K-1}{2}} \mathrm{~F}_{\rho} \frac{K-1}{2}-1\left(1+\frac{\mathrm{K}-1}{\mathrm{~N}-\mathrm{K}} \mathrm{F}_{\rho}\right)^{-\frac{\mathrm{N}-1}{2}}}{\left[\frac{\left[\frac{1}{2}(N-K)\right] !\left[\frac{1}{2}(K-1)\right] !}{\left.\left[\frac{1}{2}(N-K)\right]+\left[\frac{1}{2}(K-1)\right]-1\right]}\right.}
\end{gathered}
$$

As F-ratio $F_{\rho}$ considers residual errors $\zeta$, the Fisher's CDF $\Phi_{\mathrm{F}_{\rho}(x, K-1, N-K)}$ is reversed $\left(1-\Phi_{\mathrm{F}_{\rho}\left(\mathrm{F}_{\rho}, K-1, N-K\right)}\right)$ to generate significance score $\Phi_{\varrho}$, with greater residual errors leading to lower probability values.

This arrangement allows the decomposition of F-Ratios to the observation level, where each observation is assigned an F-Ratio value. This cumulative distribution function which follows the Fisher's Distribution serves as a score for Significance on the observation or respondent level.

Significance is an important property in DS. If income earnings are to be understood by gender and dwelling type of individuals, the latter variable provides greater noises in residual error as the separating groups in the factor create more 'noises' than the variable gender. Or the noises between age and gender are significantly different as the residual errors due to variable Age may contain more noises than the variable gender. If the 
distributions of errors among groupings are similar under the F-distribution, then the factor is less significant for use as the factor exhibits homogeneity in variances across all groups. Hence, the significance of a variable relies on the distinctiveness of errors between groups in factors, with homogeneity for within groups, or greater distances from sample mean, with lower distances from the model.

\section{Standardized Regression Coefficients as Third Property}

The third and final function of DS is the standardized parameter estimate of the regressor. This is commonly known as the slope of the curve. In a linear regression, the slope of the curve is observed by the parameter estimates of the variable $a$ of the model. However, the use of unstandardized slope of the curve is not appropriate when scales of different variables are different. Hence, the power of regressors - or 'steepness' - is then observed by the standardized parameter estimate $b$ of the model. This standardized estimate reduces the metrical scale to a common vector across all regressors. It is expressed through the following mathematical expression:

$$
\begin{gathered}
\hat{\dot{y}}=\dot{b}_{1} \theta_{1}+\dot{b}_{2} \theta_{2}+\ldots+\dot{b}_{m} \theta_{m}+\varepsilon \\
\dot{b_{m}}=b_{m}\left(\frac{\sigma_{x_{m}}}{\sigma_{y}}\right)
\end{gathered}
$$

$\theta_{i}$ denotes the standardized values of the predictor $x, \hat{\dot{y}}$ denotes the predicted response variable from standardized predictors, $\dot{b}_{m}$ denotes the standardized regression coefficient, $b_{m}$ denotes the unstandardized regression coefficient, $\sigma_{x}$ denotes the standard deviation of the predictor and $\sigma_{y}$ denotes the standard deviation of the response variable.

The absolute standardized regression coefficient $-\left|\dot{b_{m}}\right|-$ is then converted into a ratio out of the sum of all absolute standardized coefficients, with $n$ number of parameters in a model:

$$
\eta_{j}=\frac{\left|\dot{b_{j}}\right|}{\sum_{j=1}^{m}\left|\dot{b_{j}}\right|}
$$

where $m$ denotes the total number of parameter estimates in the model, $\dot{b}_{J}$ denotes the parameter estimate of interest.

While relative importance measure considers reliability and significance, power is still necessary for the understanding of relative importance of a variable in the model. When a powerful variable has low reliability and significance, a low DS score is expected. If reliability and significance scores are high, but power score is low, the DS still remains effective as the consideration that arises from the combined importance of reliability and significance outweigh the consideration for power. Intuitively, power or standardized regression coefficient is not the sole consideration of relative importance of a variable in the model but a combination of reliability, significance and power.

\section{Driver's Score (DS)}

Driver's Score (DS) is an aggregated score of three properties: reliability, significance and power. It reflects the relative importance of a variable in the model practically. At the observation level, the Drivers' Score $(\delta)$ is the geometric mean of these three properties. The mathematical expression is expressed as:

$$
\delta=\sqrt[3]{\left.\left(\frac{1}{\sigma \sqrt{2 \pi}} \int_{\min \rho}^{\rho} e^{-\left(\rho-\mu_{\rho}\right)^{2} / 2 \sigma_{\rho}^{2} d \rho \cdot\left(1-\int_{0}^{\mathrm{F}_{\rho}} \frac{\left(\frac{K-1}{N-K}\right)^{\frac{K-1}{2}} \mathrm{~F}_{\rho} \frac{K-1}{2}-1}{\left[\frac{1}{2}(N-K) !\left[\frac{1}{2}(K-1)\right] !\right.}\left[\frac{K-1}{\left[\frac{1}{2}(N-K)\right]+\left[\frac{1}{2}(K-1)\right]-1}{ }^{-\frac{N-1}{2}}\right.\right.}\right) \cdot \frac{\left|\dot{b}_{j}\right|}{\sum_{j=1}^{m}\left|\dot{b}_{j}\right|}\right)}
$$

As observed in the DS equation, the DS methodology is the cube-root of the ratio of three products to the sum of 
all absolute standardized regression coefficient estimates. It is a function of Reliability (Gaussian's CDF which has an exponential function), Significance (Fisher's CDF which has a function of the hypergeometric function for $F_{\rho}$ by Beta function for $F_{\rho}$ ) and the absolute standardized regression coefficient of the variable of interest and an inverse function of the sum of all absolute standardized regression coefficient estimates. The decrease in other absolute standardized parameter estimates increases DS. This understanding has an important and practical value: the variable of interest in which it is positioned with other variables is influenced by the mix of the model.

When DS is low, either two of the three properties or all properties are low, resulting to weaker drivers' influence. When DS is high, either two of the three properties or all properties are high, resulting to stronger drivers' influence. DS is bounded by a lower limit and an upper limit of 0 and 1 respectively.

At the regressor level, the Drivers' Score (DS) is the arithmetic mean of all the geometric mean of all three properties at the observation level:

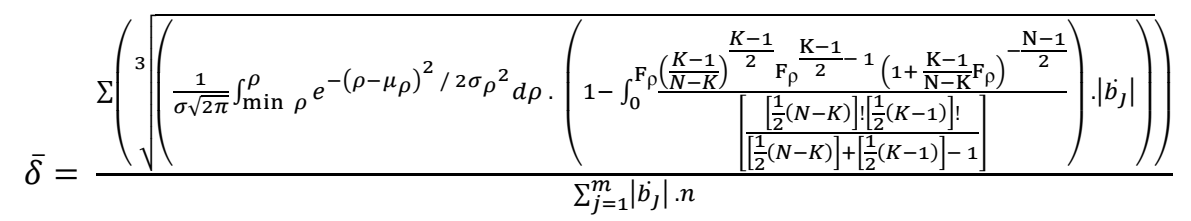

When DS at the regressor level is low, relative influence on the dependent variable in the model is low. When DS at the regressor level is high, relative influence on the dependent variable in the model is high. DS is bounded by a lower limit and an upper limit of 0 and 1 respectively.

\section{Weighted Drivers' Score (wDS)}

The weighted Drivers' Score (wDS) is a composite score of three properties (RSP), being weighted individually by their importance in the DS framework. For example, the score for reliability (R) is far more important than the score for significance (S) and power (P). Hence, the R score is given heavier weights than the rest. The wDS - $\delta_{w}$ - can be expressed mathematically by assigning power to the properties:

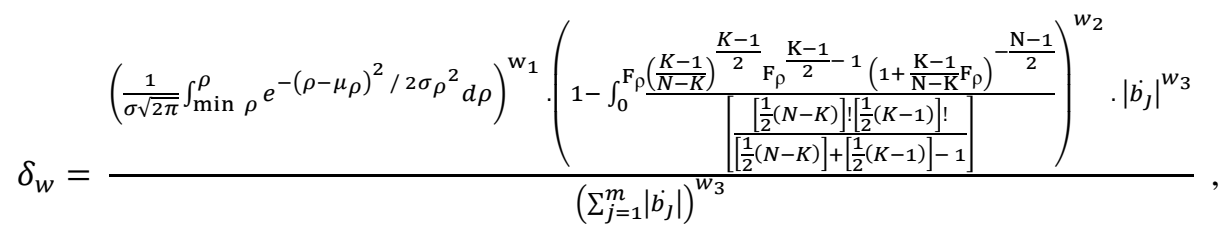

$$
\begin{aligned}
& w_{1}+w_{2}+w_{3}=1
\end{aligned}
$$

At the regressor level, the weighted Drivers' Score which is relative importance of a variable in the model after adding weights is the arithmetic mean of all three properties at the observation level:

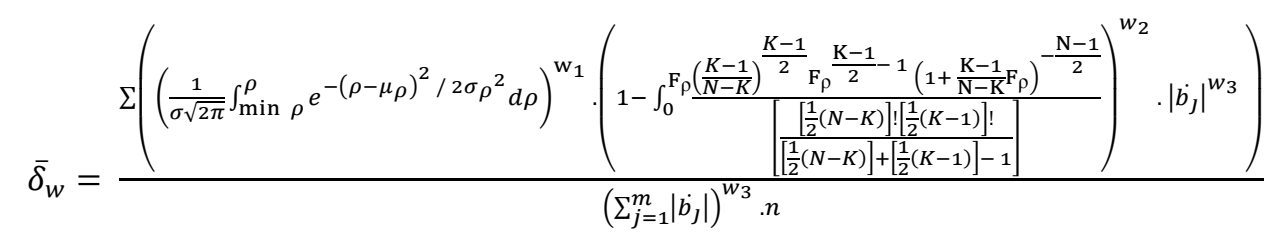

\section{Application of DS}

Using data from a Multinational Corporation (MNC) that is based in Singapore (Note 1), the spread of DS of 4 predictors and its' response variable are: 


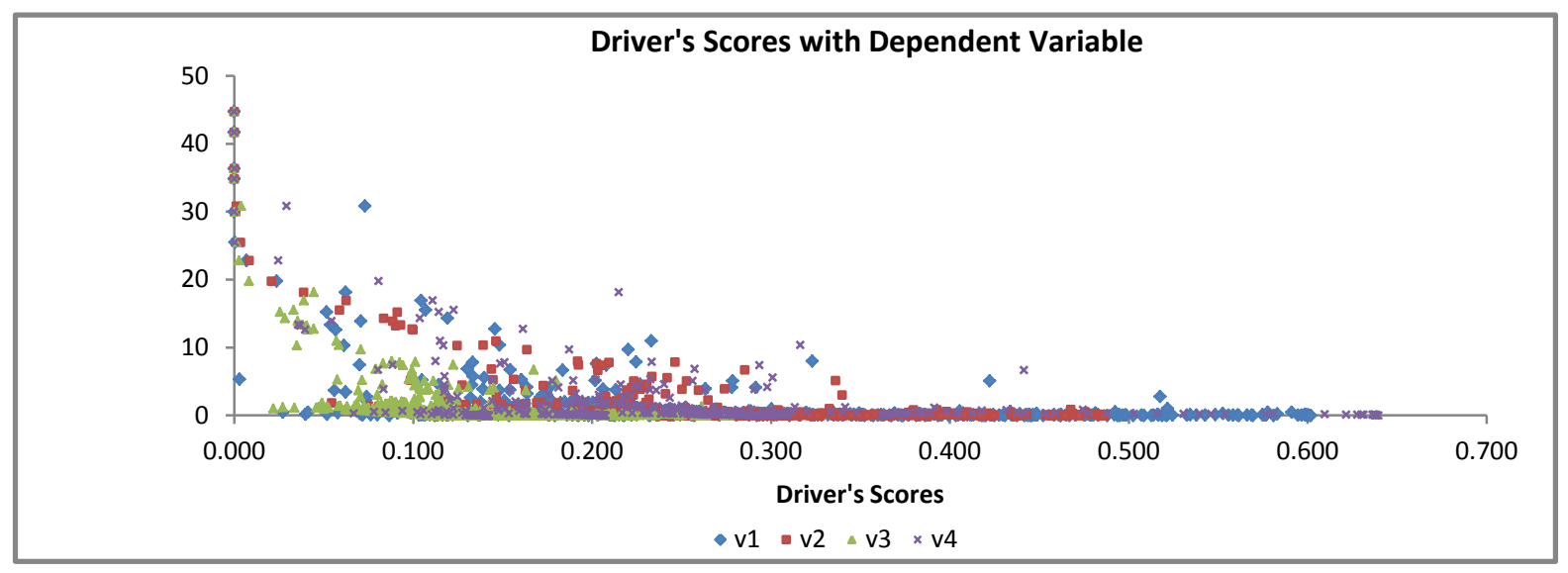

Figure 1. DS of v1 to v4 on dependent variable

Based on insights that were gathered from professionals in the industry, $v 1$ has the greatest impact or driving force in understanding the dependent variable. This impact or driving force is understood under the framework of the RSP model. To garner reliable, significant and powerful dependent variable, extra care must be given to $v 1$.

As it is observed from the chart above (Figure 1), the relationship between DS and its dependent variable takes on the inverse exponential function. This would explicitly highlight that the observations of RSP is often the inverse of the dependent variable. The tendency to mispredict is higher when dependent variable or its set of products gets too complex. In this area, the DS model has intuitively performed well.
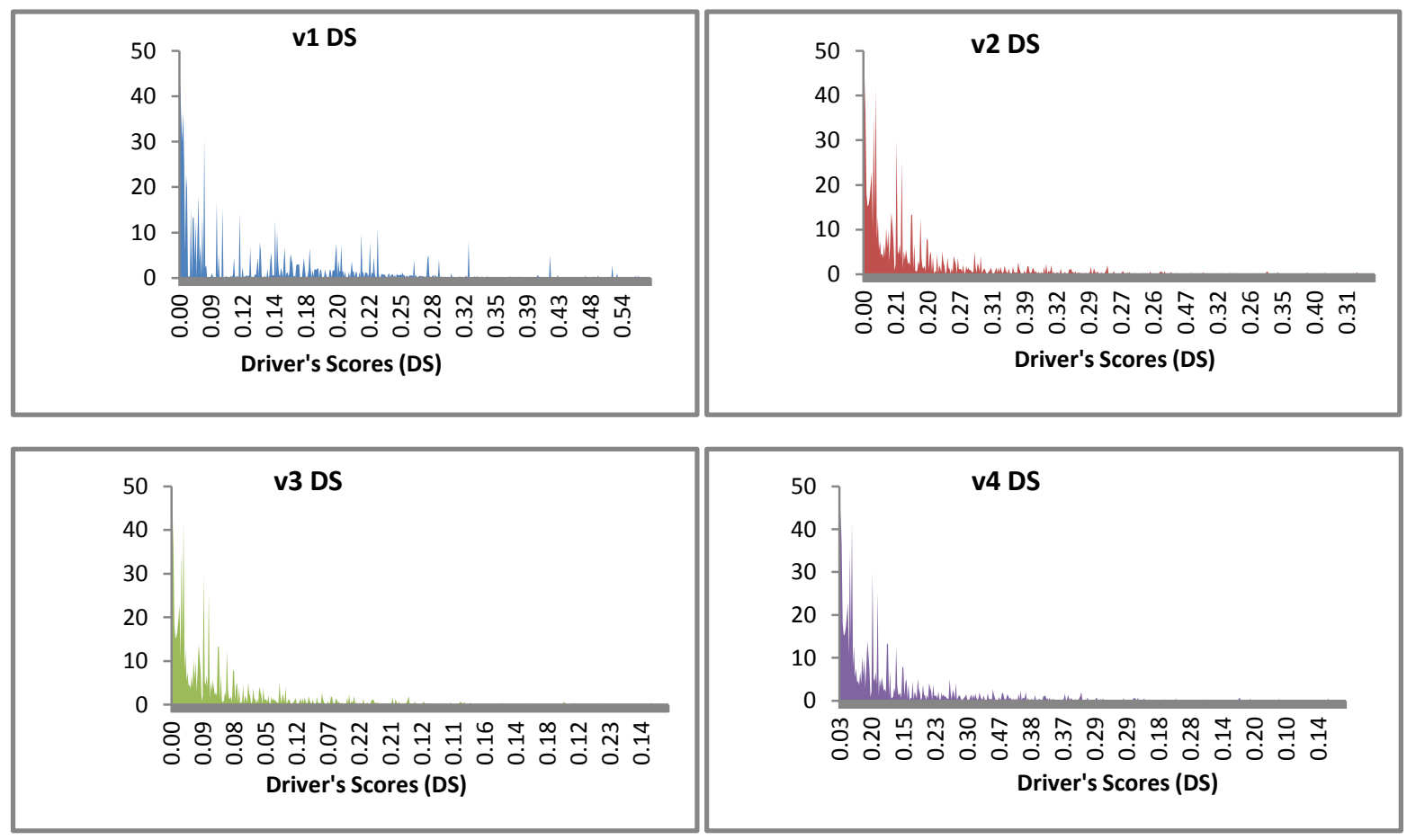

Figure 2. Scatter-plots of DS with Dependent Variable

The plots in figure 2 show the area under the curve, which represents the magnitude of DS. VI has the largest area under the curve.

\section{VIF and Its Decomposition}

The relative importance of $j$ variable $-\bar{\delta}$ - is the arithmetic mean of summation of all $\delta$ in $j$ variable: 


$$
\begin{gathered}
\delta_{i, j}=\sqrt[3]{\Phi_{\left(\rho_{i, j}\right)} \cdot \Phi_{\mathrm{F}_{\rho}\left(\mathrm{F}_{\rho}, K-1, N-K\right)} \cdot \eta_{j}} \\
\bar{\delta}_{j}=\frac{\sum_{i=1}^{n} \delta_{i, j}}{n}
\end{gathered}
$$

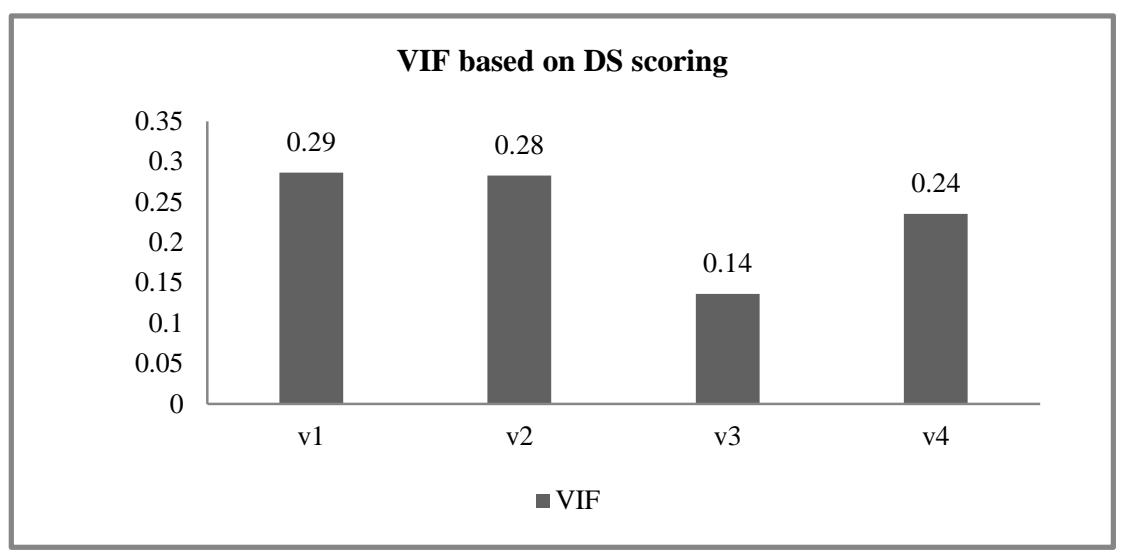

Figure 3. VIF chart

As observed in the bar chart, the strongest relative importance of factor in the model is $v 1$. This importance is decomposed into its RSP properties, which is shown in the table below:

Table 1. Average RSP and DS Scores against Four Independent Variables

\begin{tabular}{ccccc}
\hline & $\boldsymbol{R}$ & $\boldsymbol{S}$ & $\boldsymbol{P}$ & $\boldsymbol{D}$ \\
\cline { 2 - 4 } $\boldsymbol{v 1}$ & 0.5454 & 0.2148 & 0.3540 & 0.2867 \\
$\boldsymbol{n} 2$ & 0.5481 & 0.3276 & 0.1830 & 0.2829 \\
$\boldsymbol{v 3}$ & 0.5447 & 0.2067 & 0.0340 & 0.1363 \\
$\boldsymbol{v 4}$ & 0.5364 & 0.1131 & 0.4290 & 0.2353 \\
\hline Mean & 0.5437 & 0.2156 & 0.2500 & 0.2353 \\
\hline
\end{tabular}

Note. The DS score is the geometric mean of all three RSP scores.

In this example, the use of the standardized parameter estimate - $\boldsymbol{P}$ - as the relative influence factor is misleading as industry practitioners have clearly identified the importance of $v l$ rather than $v 4$. This is also reflected in the DS score. The other use of variance decomposition $-\boldsymbol{R}$ - is also misleading, as it identifies $v 2$ as the factor that has the strongest relative importance in the model instead of $v 1$. The composite score of three properties clearly show that variable importance does not solely rely on one aspect or dimension of importance considerations, but three aspects: reliability, significance and power.

A 2x6 Variable Importance Matrix (VIM) describes the characteristics of DS. A low score is understood as a score that is below 0.50. A high score is understood as a score that is above 0.50 . For example, a mix of DS=0.90 (highest DS score in the model) and $\mathrm{R}=0.30$ is characterized as the most important variable that has low reliability. From the VIM table, this variable is known as an Unreliably Important variable.

Table 2. Variable Importance Matrix (VIM)

\begin{tabular}{|c|c|c|}
\cline { 2 - 3 } \multicolumn{1}{c|}{} & \multicolumn{1}{c|}{ Less Important } & Most Important \\
\hline Low Reliability & Unreliably Trivial & Unreliably Important \\
\hline High Reliability & Reliably Trivial & Reliably Important \\
\hline Low Significance & Insignificantly Trivial & $\begin{array}{c}\text { Insignificantly } \\
\text { Important }\end{array}$ \\
\hline High Significance & Significantly Trivial & $\begin{array}{c}\text { Significantly } \\
\text { Important }\end{array}$ \\
\hline Low Power & Powerlessly Trivial & $\begin{array}{c}\text { Powerlessly } \\
\text { Important }\end{array}$ \\
\hline High Power & Powerfully Trivial & Powerfully Important \\
\hline
\end{tabular}


A classification by levels (CLASS) is used here:

If ALL RSP is low (i.e. $R S P<0.50) \Rightarrow$ Level 4

If EITHER Two of RSP is low (i.e. $R P / R S / S P<0.50) \Rightarrow$ Level 3

If ONLY One of RSP is low (i.e. $R / S / P<0.50) \Rightarrow$ Level 2

If NONE of RSP is low (i.e. $R S P \geq 0.50) \Rightarrow$ Level 1

The CLASS logic is then combined with VIM to generate the final descriptive outcome of VIF for the variable of interest. For example, $v l$ is classified as level 3 Importance (L3I) variable. However, DS can be improved if the CLASS level improves from level 3 to level 1. Ideally, a good and strong driver for response variable is a level 1 importance (L1I) variable. To achieve such a score, RSP scores that are below 0.50 should be given extra attention. For example, $v l$ has high $\mathbf{R}$ score but low $\mathbf{S}$ and $\mathbf{P}$ scores. Hence, measures to create greater distinctiveness and power could potentially improve the final DS score.

The benefit of this scoring methodology is 1) the flexibility to include categorical variables as a regressor, 2) the ability to aggregate three important properties of variable from both the theoretical and practical aspects of analytic, and 3) the simplicity at which an explanation could be given to laypeople.

Categorical variables are treated as dummy variables in the linear regression model and its' F-ratios are similarly calculated by taking the sum of squared error due to model against the sum of squared error due to residual errors. To assign power to the DS methodology, absolute standardized regression coefficients for each dummy variable is assigned to each groupings in the factor. Hence, a metrical independent variable has one power assignment in a factor while the categorical independent variable has multiple power assignments for different groupings in a single factor.

Theoretically, so long as the variable is normally distributed and conforms to the chi-square distribution, the usefulness of the DS methodology becomes essential for practical use. A single dimension of the DS methodology may be taken to generate VIF measurement, but it does not represent the natural and intuitive considerations of factor importance in practice. Practitioners often need a score that reflects reliability, significance and power of the predictors and conclude the importance of it thereafter. DS helps to meet the needs of those who wish to obtain a practical assessment of variable importance.

\section{Limitations of DS}

DS has several limitations. Firstly, DS takes the absolute standardized regression coefficients as a measurement for power in a linear model. In cases where nonlinearity is observed, an alternative approach is needed to substitute this third property of DS. Also, DS does not account for multi-dimensional explanation of variances in a single vector space. This has implications when it comes to interrelatedness of predictors that could potentially form interaction terms. When interaction terms are formed, the DS methodology is not able to tease out interaction effects. Lastly, as categorical variables are converted into dummy variables in a model, the increase in parameter estimates with constant set of sample size makes the model unstable. Hence, the DS methodology becomes useful when only key factors that could potentially explain variances better are considered. These limitations have an impact in practical use when the relationship between predictors and response variable becomes too complex, resulting to multiple unknown 'noises' within the model that could affect the whole DS methodology. The use of DS should be jealously guarded by good and firm understanding of relationship between variables and the intuitive approach in understanding practical relationships is essential in the DS methodology.

\section{Summary}

Managers often ask what drivers or variables influence the outcome of success more significantly over the other at the respondent level. For example, the main question that managers ask is the drivers which contribute to the success of an event. Fundamentally, the drivers that contribute mostly to success of an event are obtained by the correlation coefficient of the variables. However, this methodology falls apart when categorical variables are used or the requirement to assess additional parameters is apparent. DS is designed to overcome this limitation by decomposing the DS scores into its three properties - RSP. As the measurement for variable influence is seen at the observation or respondent level, the aggregation of scores can finally add up to the variable influence factor (VIF) measurement.

Cost-cutting measures that lead to improved profitability are also possible by using DS. Businesses can utilize DS to determine which dimension or variable has the greatest importance and pitch marketing efforts on that dimension with a targeted focus on respondents who have higher RSP scores. If the amount of sales leads is a 
stronger or more important factor in determining revenue and the higher RSP scores are found among the entrepreneurs, then companies can focus their marketing effort on generating leads by targeting the entrepreneurs first, then the others. Although it is fundamentally true that the outcome of revenue takes prerogative in marketing effort, the precision of the outcome and the significance of it have a key role to play in understanding variable importance. Targeted and focused marketing can generate income faster, which could then supplement additional capital to target the rest of the populations using low-cost marketing efforts.

\section{Future Research}

Due to the limitation imposed by the DS methodology, more studies can be conducted to understand how variance decomposition occurs in a multi-dimensional setting in a single vector space. Particularly, studies can be conducted to examine independent variables that are correlated to each other and when these variables are categorical, more can be done to examine interaction terms and how it decomposes to the RSP scorings. Finally, more 'real-world' data is needed to assess the DS methodology, particularly in RSP scorings. A survey of factor importance can be distributed to industry practitioners to complement the findings of DS. The results from the survey can validate the strength of RSP scoring methodology. While the RSP scoring and its DS methodology is still at its conceptual stage, the use of it can bring many benefits to business practitioners and academic researchers. In light of the possible use of DS, more industrial report can utilize this RSP scoring method to generate analytic-based decision makings.

\section{References}

Chevan, A., \& Sutherland, M. (1991). Hierarchical Partitioning. The American Statistician, 90-96. http://dx.doi.org/10.2307/2684366

Christensen, R. (1992). Comment on "Hierarchical Partitioning," by A. Chevan and M. Sutherland. The American Statistician, 74.

Darlington, R. (1968). Multiple Regression in Psychological Research and Practice. Psychological Bulletin, 161-182. http://dx.doi.org/10.1037/h0025471

Ehrenberg, A. (1990). The Unimportance of Relative Importance. The American Statistician, 260.

Hoffman, P. (1960). The Paramophic Representation of Clinical Judgment. Psychological Bulletin, 116-131. http://dx.doi.org/10.1037/h0047807

Hooker, R., \& Yule, G. U. (1906). Note on Estimating the Relative Influence of Two Variables Upon a Third. Journal of the Royal Statistical Society, 197-200. http://dx.doi.org/10.2307/2339552

Kruskal, W. (1987). Relative Importance by Averging Over Orderings. The American Statistician, 6-10.

Lindeman, R., Merenda, P., \& Gold, R. (1980). Introduction to Bivariate and Multvariate Analysis. Glenview: Scott, Foresman.

Pratt, J. W. (1987). Dividing the Indivisible: Using Simple Symmetry to Partition Variance Explained. Proceedings of Second Tampere Conference in Statistics, eds. (pp. 245-260). Finland: T. Pukkila and S. Puntamen.

Strobl, C., Boulesteix, A., Kneib, T., Augustin, T., \& Zeileis, A. (2008). Conditional Variable Importance for Random Forests. BMC Bioinformatics , 307. http://dx.doi.org/10.1186/1471-2105-9-307

Stufken, J. (1992). On Hierarchical Partitioning. The American Statistician , 70-71.

Note 1: With permission granted from the Multinational Corporation (MNC), under the condition of full anonymity and non-disclosure.

\section{Copyrights}

Copyright for this article is retained by the author(s), with first publication rights granted to the journal.

This is an open-access article distributed under the terms and conditions of the Creative Commons Attribution license (http://creativecommons.org/licenses/by/3.0/). 